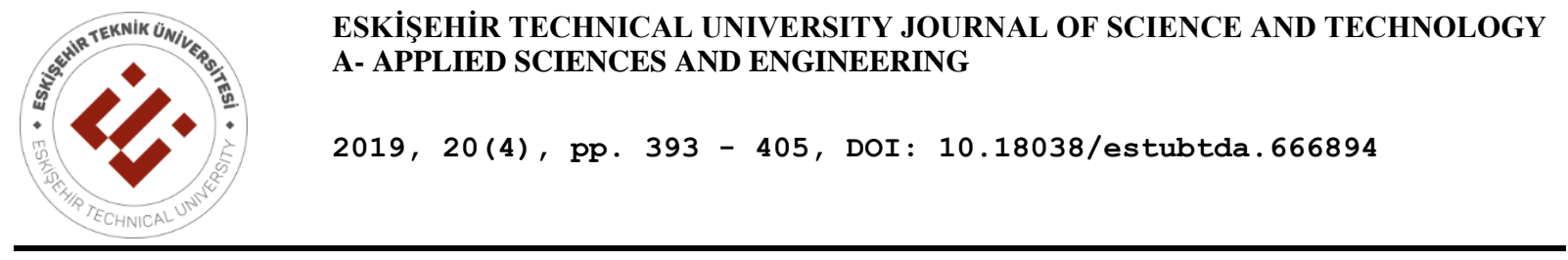

\title{
THE PHYSICOCHEMICAL CHARACTERIZATION AND KINETIC PARAMETER ASSESSMENT OF HYDROXYAPATITE CRYSTALS
}

\author{
Sevgi POLAT* \\ Department of Chemical Engineering, Faculty of Engineering, Marmara University, 34722, İstanbul, Turkey
}

\begin{abstract}
The crystallization of hydroxyapatite (HAP) was analyzed in a batch system in pure medium and in the presence of butyric acid as an additive. The experiments were conducted at different additive concentrations and the influences of the additive were investigated. Firstly, the crystals obtained were characterized by X-ray diffraction, Brunauer-Emmett-Teller, transmission electron microscopy, zeta potential measurement, Fourier transform infrared spectroscopy, and thermogravimetric analysis. The structure, surface areas, morphology, surface potential charges, functional groups and thermal decomposition behavior of the crystals were determined. In the next step, thermal decomposition kinetic profiles were modeled using the distributed activation energy model (DAEM), Flynn-Wall-Ozawa (FWO) and Friedman models. All the models used provided accurate fits of the TGA data with acceptably high $\mathrm{R}^{2}$ values. The value for the crystals obtained from the butyric acid containing medium was about $589 \mathrm{~kJ} / \mathrm{mol}$ higher than that found for the crystals from the pure medium $(538 \mathrm{~kJ} / \mathrm{mol})$. According to the results of the characterization and kinetic analysis, butyric acid could be employed as an additive for the production of HAP crystals with the desired quality and physical properties.
\end{abstract}

Keywords: Hydroxyapatite, Crystallization, Kinetics, Butyric acid

\section{INTRODUCTION}

The precipitation and dissolution of sparingly soluble calcium phosphate salts have captured the attention of numerous investigators, mainly because of their role in biological calcification processes $[1,2]$. As the primary inorganic component of bones and teeth, the most stable calcium phosphate salt among the calcium phosphate phases is hydroxyapatite $\left[\mathrm{Ca}_{10}\left(\mathrm{PO}_{4}\right)_{6}(\mathrm{OH})_{2}, \mathrm{HAP}\right]$. Thanks to its chemical and structural resemblance to bone minerals and excellent biocompatibility, it is also broadly used in various biomedical fields from dental composites [3] and bone tissue engineering [4] to orthopedic implants [5]. Its wide-ranging uses have sparked interest in synthetic, extra pure, and welldefined HAP crystals for use in detailed physicochemical studies, medical applications, and surgery [6-8]. Several studies have reported drastic changes in the nucleation rate, growth rate, and morphology of HAP crystals with the addition of even small amounts of additives. Koutsopoulos and Dalas [9] researched the impacts of aspartic and glutamic acids on hydroxyapatite crystallization. They found that both acids, when used as additives, decreased the HAP crystal growth rate by blocking of the active growth sites on the HAP crystals' surface. Koutsopoulos et al. [10,11] investigated the role of various type of amino acids, such as serine, tyrosine, hydroxyproline, lysine, cysteine, cystine, and glutamine. They detected that these amino acids caused reductions in the crystal growth of HAP. Oner and Dogan [12] examined the effects of polyelectrolytes on HAP crystallization kinetics. They found that polyelectrolytes blocked the crystal growth rate and the average particle size of the crystals decreased in their presence. Another study conducted by Mangood et al. [13] demonstrated that the growth rates of HAP crystals decreased in the presence of acetaminophen, but no changes were observed in the morphology of the crystals. Lima et al. [14] investigated HAP nanocrystals in the presence of chromium (III) ions. The results showed that the crystals doped with chromium experienced morphological changes and the crystals obtained had an agglomerated rod-like morphology. Bucur et al. [15] researched the influence of tartaric acid on HAP crystal morphology.

*Corresponding Author: sevgi.polat@marmara.edu.tr

Received: 11.10.2018 Published: 30.12.2019 
Scanning and transmission electron microscopy (SEM and TEM) results indicated that the addition of tartaric acid at low concentration affected the morphology of the crystals. All of these studies reported changes in certain characteristics of HAP crystals with the addition of additives, which included changes in their morphology, size, and degree of crystallinity. Through these changes, the physical and chemical properties of synthetic HAP can be controlled for use in specific biomedical applications [12]. For this purpose, the effects of the use of butyric acid, a type of carboxylic acid used in the pharmaceutical, chemical and food industries [16], as an additive to HAP crystallization were investigated in this study. A better understanding of the effect of butyric acid in the HAP structure would provide useful information for improving the physical properties of the HAP crystals. Moreover, the effects of butyric acid on the thermal decomposition behaviour were investigated and the activation energy of the crystals obtained from the additive containing and non-additive media was calculated using different kinetic methods.

\section{EXPERIMENTAL METHOD}

\subsection{Materials}

All chemicals used in the experiments were of analytical grade. Calcium chloride $\left(\mathrm{CaCl}_{2}\right)$, potassium dihydrogen phosphate $\left(\mathrm{KH}_{2} \mathrm{PO}_{4}\right)$, and butyric acid $\left(\mathrm{C}_{4} \mathrm{H}_{8} \mathrm{O}_{2}\right)$ were obtained from Merck. Triply distilled $\mathrm{CO}_{2}$-free water was used in all experiments.

\subsection{Crystallization Experiments}

The experiments were done in a double-jacketed crystallizer with an active volume of $1 \mathrm{~L}$. The experimental setup is shown in Figure 1.

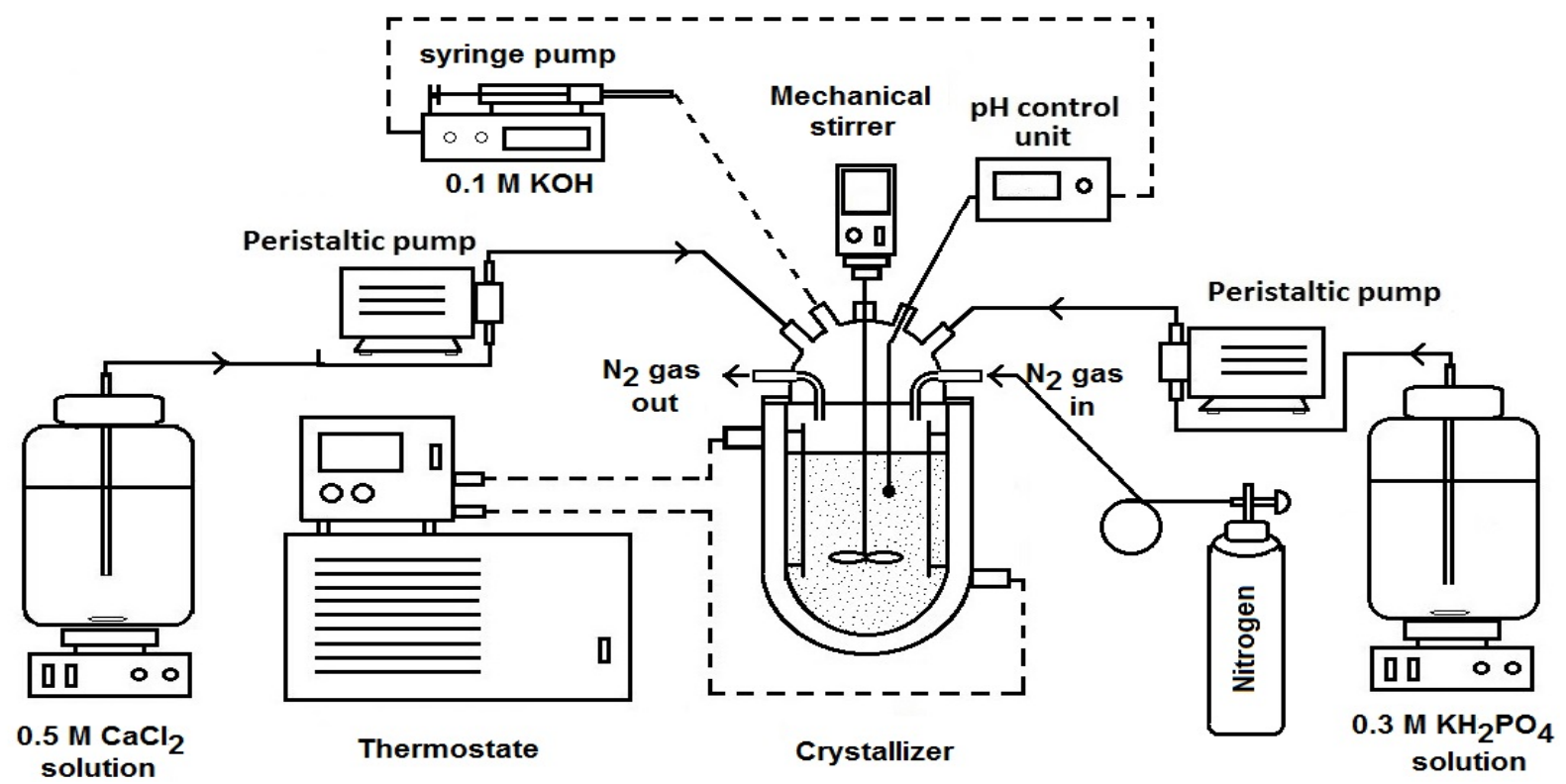

Figure 1. Experimental set-up

Initially, the crystallizer was loaded with $500 \mathrm{~mL}$ of $\mathrm{CO}_{2}$-free triply distilled water and allowed to reach thermal equilibrium at $70^{\circ} \mathrm{C}$. The internal temperature of the crystallizer was kept constant at $70 \pm 0.5$ ${ }^{\circ} \mathrm{C}$ through a thermostat. Solutions of $0.5 \mathrm{M} \mathrm{CaCl}_{2}$ and $0.3 \mathrm{M} \mathrm{KH}_{2} \mathrm{PO}_{4}$ solution were fed to the crystallizer via peristaltic pumps at a rate of $8 \mathrm{~mL} / \mathrm{min}$. The $\mathrm{pH}$ in the crystallizer was kept constant at 9.5 by means of a $\mathrm{pH}$ control system. A $0.1 \mathrm{M} \mathrm{KOH}$ solution was used for $\mathrm{pH}$ adjustment. A three-blade 
propeller type stirrer element was used and the stirrer speed was set at $600 \mathrm{rpm}$. During the experiment, the lid of the crystallizer was kept closed to prevent ingress of $\mathrm{CO}_{2}$ from the environment and $\mathrm{N}_{2}$ gas was fed into the crystallizer above the liquid level. The suspension obtained at the end of the experiment was separated from the solution and the obtained HAP crystals were washed with $\mathrm{CO}_{2}$-free distilled water until no chloride ions remained. The HAP crystals completely free of chlorine ions were placed in Erlenmeyer flasks and stored for 2 months in a water bath at a pH of 7.4 and a temperature of $37^{\circ} \mathrm{C}$. At the end of this process, the crystals were filtered and dried in a vacuum oven. The HAP crystals thus obtained were characterized using various characterization techniques. Butyric acid was used as an additive and was applied at concentrations of 50,100, and $200 \mathrm{ppm}$. The additive was fed into the crystallizer with an infusion pump in a synchronous manner with the peristaltic pumps so that the desired concentration could be obtained. The same operational conditions as those used for the pure medium were applied for the experiments conducted in the presence of the additive. Moreover, the same experimental procedure, including analysis, was also applied to the experiments in which the additive was used.

\subsection{Characterization of the Crystals}

First, X-ray diffraction (XRD) analysis was performed to determine the crystal structure using a Bruker D2 Phaser table-top diffractometer. The device was equipped with $\mathrm{Cu} \mathrm{K}$ radiation (30 kV) and a step size of 0.01 . The patterns were recorded for $2 \theta$ values of 10-600. TEM and Brunauer-Emmett-Teller (BET) analysis were used to detect the morphological changes and the crystal surface area, respectively. BET analysis was performed using Quadrasorb SI (Quantachrome) analyzer and the samples were previously degassed for $2 \mathrm{~h}$ at $200^{\circ} \mathrm{C}$ under vacuum. The surface charge of the crystals was detected by zeta potential measurement and the analysis were carried out using a Malvern Zetasizer Nano ZS instrument with 8-10 measurements and a total of 50 runs per sample. The functional groups of crystals produced in non-additive and additive medium were characterized by Fourier transform infrared spectrometry (FTIR) analysis. FTIR spectra were scanned between 4000 and $650 \mathrm{~cm}^{-1}$ using a Perkin Elmer Spectrum 100 model Fourier Transform Infrared Spectrometer. In addition, the thermal degradation behaviors of the crystals were determined using thermogravimetric and differential thermogravimetric (TGA/DTG) analysis. Thermal analysis was performed in the temperature range of $30-1250{ }^{\circ} \mathrm{C}$ with different heating rates of 5,10 and $20^{\circ} \mathrm{C} / \mathrm{min}$ under a nitrogen atmosphere at a flow rate of $20 \mathrm{~mL} / \mathrm{min}$ by Setaram LABSYS Evo Thermal Analyzer. At the same time, kinetic calculations were also carried out using the TGA data.

\section{RESULTS and DISCUSSION}

\subsection{Characteristics of the Crystals}

The HAP crystals acquired in pure solution and solutions containing different concentrations of butyric acid were characterized by various methods to determine the influence of butyric acid addition on the physical properties of the crystals.

Firstly, the XRD spectra of the HAP crystals obtained in pure medium and the presence of butyric acid are given in Figure 2. The XRD patterns were compared with the data given in the literature [17]; HAP crystals were obtained in all conditions and no different phase formation was observed. Compared to the pure medium, an increase in peak intensities was seen with the addition of butyric acid to the crystallization medium. In addition, there was a slight shift in the peak values measured at a high angle with increasing additive concentration. 


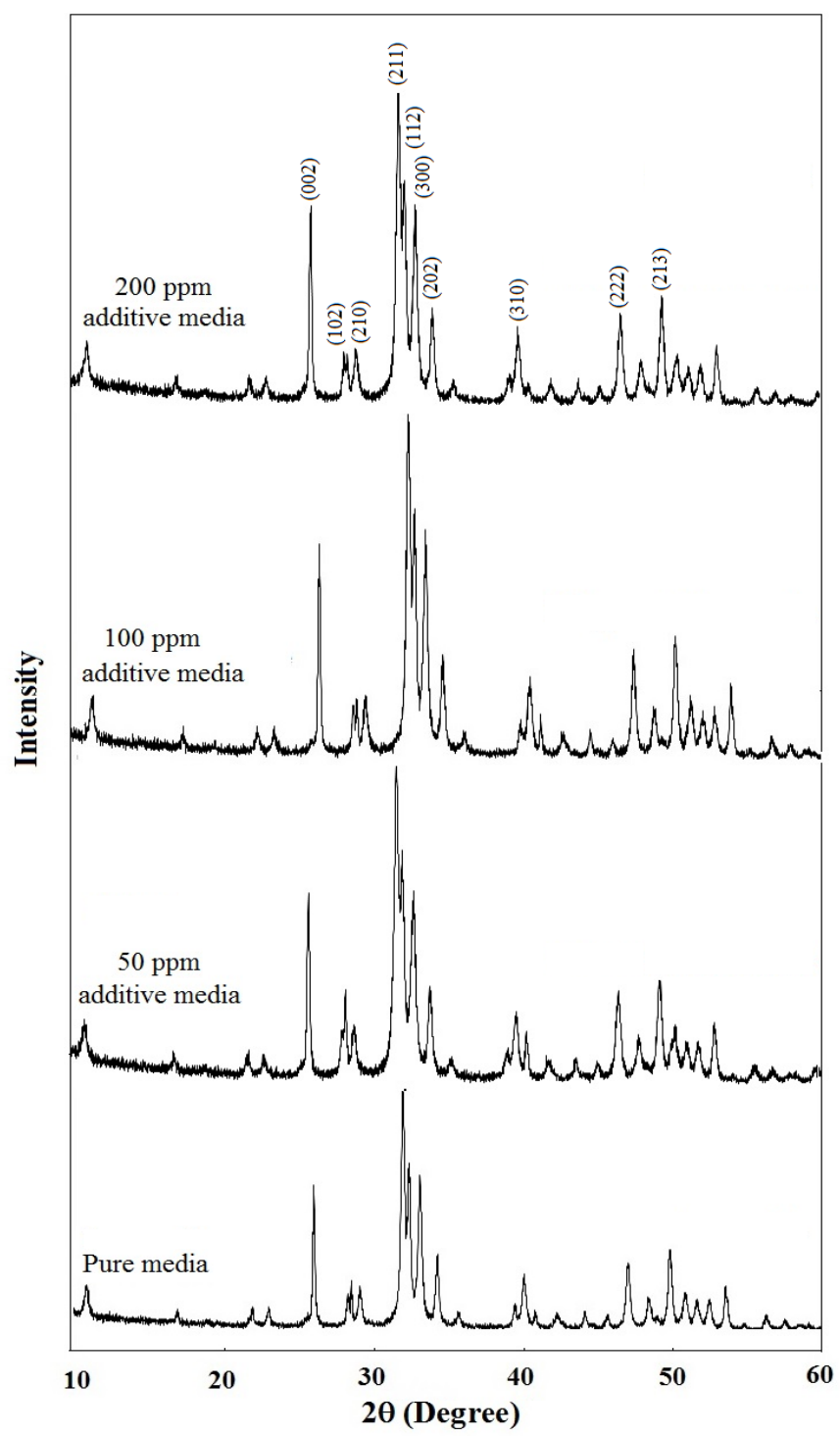

Figure 2. XRD spectra of the crystals acquired in pure medium and medium containing different concentrations of butyric acid.

The information about the crystal morphology was provided by TEM analysis. TEM images of the crystals obtained in pure solution and the solutions with three different concentrations of butyric acid are given in Figure 3. The HAP crystals obtained from the non-additive medium had a clear rod-like morphology. The crystals tended to grow on each other and had an agglomerated appearance. This result was similar to previous studies in the literature $[18,19]$. HAP crystals formed in the presence of $50 \mathrm{ppm}$ additive were in a rod-like form and they kept their stick forms and the tips of the crystals had a more sharp view. At the same time, surface nucleation began to appear on the crystal surface with increasing concentration of the additive. An agglomeration effect was widely seen in the crystals obtained from the crystallization medium containing $100 \mathrm{ppm}$ butyric acid. There was an increase in the dimensions of the crystals relative to those prepared in pure medium. A significant increase was observed in the aspect ratio of the crystals and the size of the crystals also increased further. In the presence of $200 \mathrm{ppm}$ of additive, the increasing size tendency observed continued and agglomeration also appeared more frequently at this concentration. Compared to pure medium, it was clear that butyric acid had an effect on the crystal morphology and crystal size. 
Polat / Eskişehir Tech. Univ. J. of Sci. and Technology A - Appl. Sci. and Eng. 20 (4) - 2019
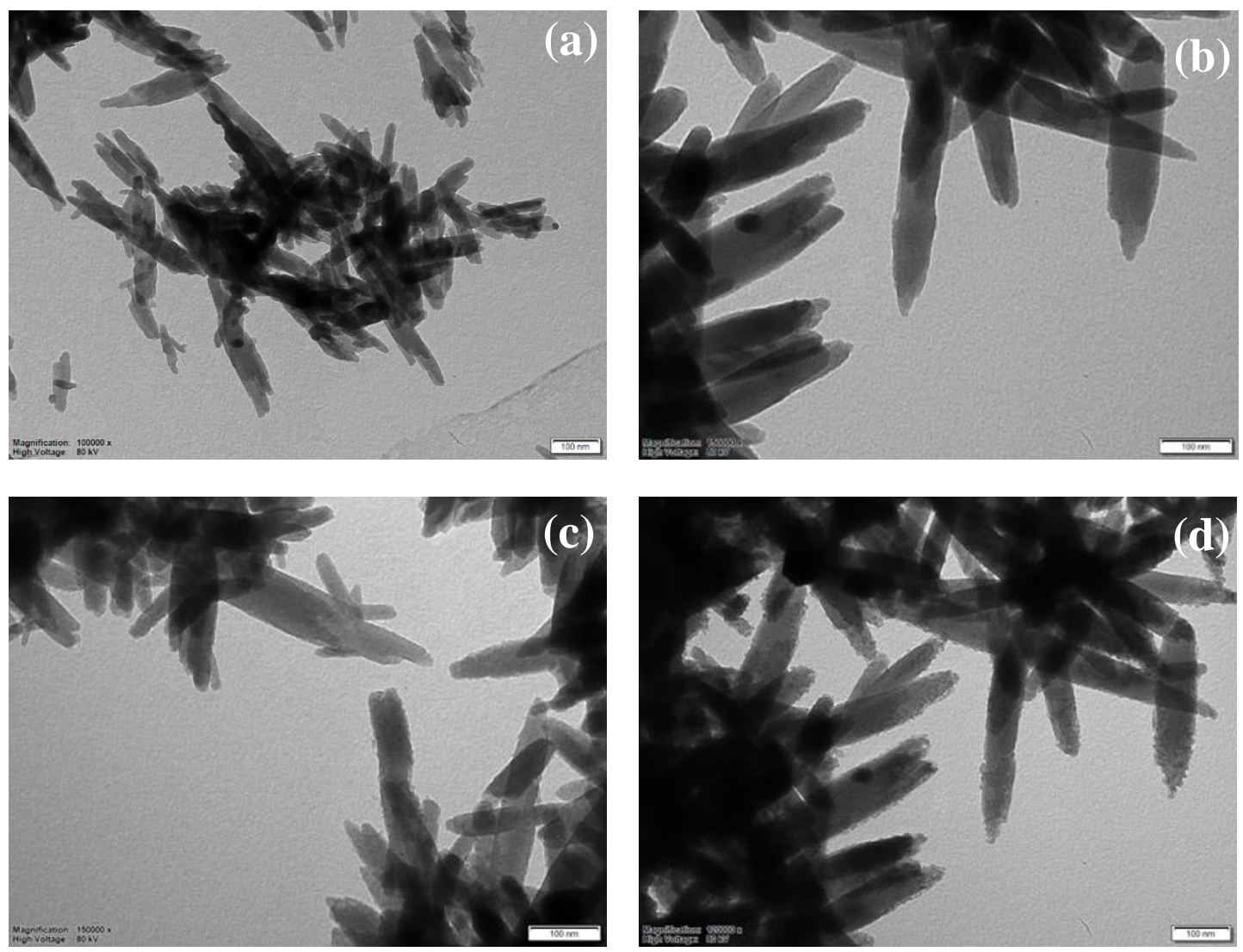

Figure 3. The TEM images of the crystals acquired a) in pure medium and medium containing butyric acid at b) $50 \mathrm{ppm}$, c) $100 \mathrm{ppm}$, and d) $200 \mathrm{ppm}$.

The BET surface areas of the HAP crystals obtained from the additive containing and non-additive media were measured to determine what the effect of the additive on the surface area of the crystals. The results are presented in Figure 4.

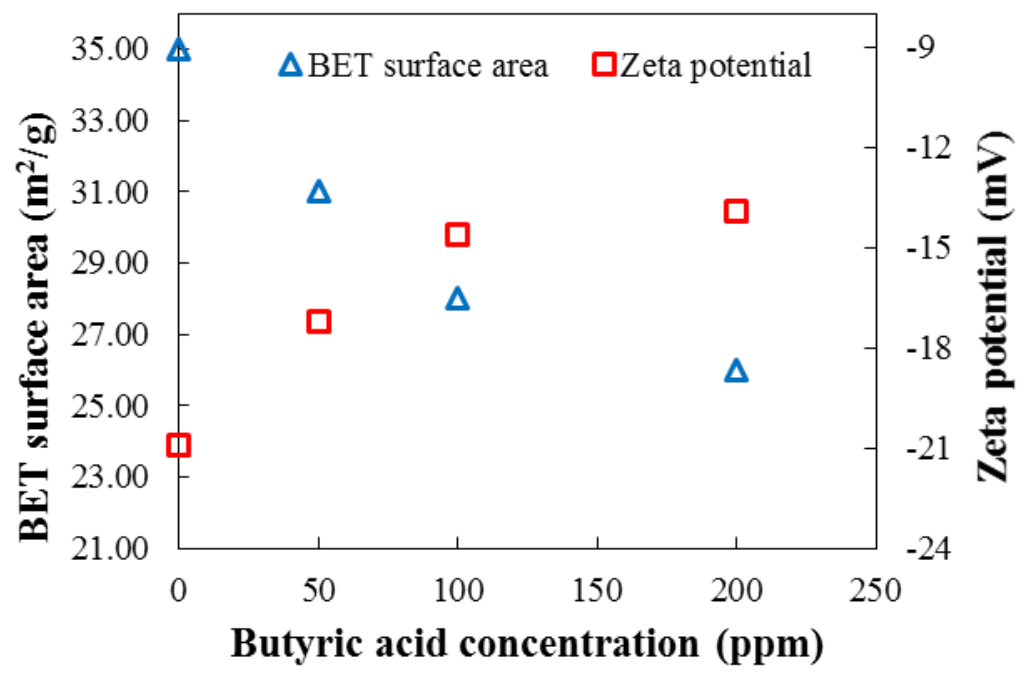

Figure 4. The variation of BET surface areas and zeta potentials of the HAP crystals as a function of butyric acid concentration. 
The BET specific surface area of pure HAP crystals was $35 \mathrm{~m}^{2} / \mathrm{g}$. This result was in good agreement with previous studies [12]. This measured value reduced as the butyric acid concentration increased. The specific surface areas of the crystals acquired in solutions containing 50, 100, and $200 \mathrm{ppm}$ concentrations of butyric acid were measured as 31,29 , and $26 \mathrm{~m}^{2} / \mathrm{g}$, respectively. The variations in the measured values were related to the crystal size change due to the additive effect. The addition of the additive to medium also led to the decreasing the pore volume of the crystals. While the cumulative adsorption volume of the pores for the crystals acquired from the pure solution was $0.005 \mathrm{~cm}^{3} / \mathrm{g}$, this value decreased to $0.003 \mathrm{~cm}^{3} / \mathrm{g}$ in the presence of $200 \mathrm{ppm}$ additive medium. From the results, it was noticeable that the presence of butyric acid led to a lower specific surface area and reduced the pore volume of the HAP crystals.

Zeta potential analysis was performed for the crystals obtained in pure solution and solutions containing various butyric acid concentrations to determine the changes that occurred in the surface potential of HAP nanocrystals. The results are shown in Figure 4. The zeta potential of HAP crystals obtained in non-additive medium at a $\mathrm{pH}$ of 7.4 was measured as $-20.9 \mathrm{mV}$, which was similar to previous studies on HAP crystals in neutral conditions [20]. The surface charges of the HAP crystals changed with the addition of butyric acid to the medium and became less negative as the additive concentration increased. For 50, 100, and $200 \mathrm{ppm}$ additive concentrations, these values were measured as -17.2, -14.6, and $13.9 \mathrm{mV}$, respectively. This reduction in the negativity can be explained by the interaction between HAP and butyric acid.

FTIR analysis was performed for the crystals obtained from the pure solution and the solution containing additive to investigate the binding of butyric acid to the HAP surface. The FTIR spectra of the HAP crystals are given in Figure 5.

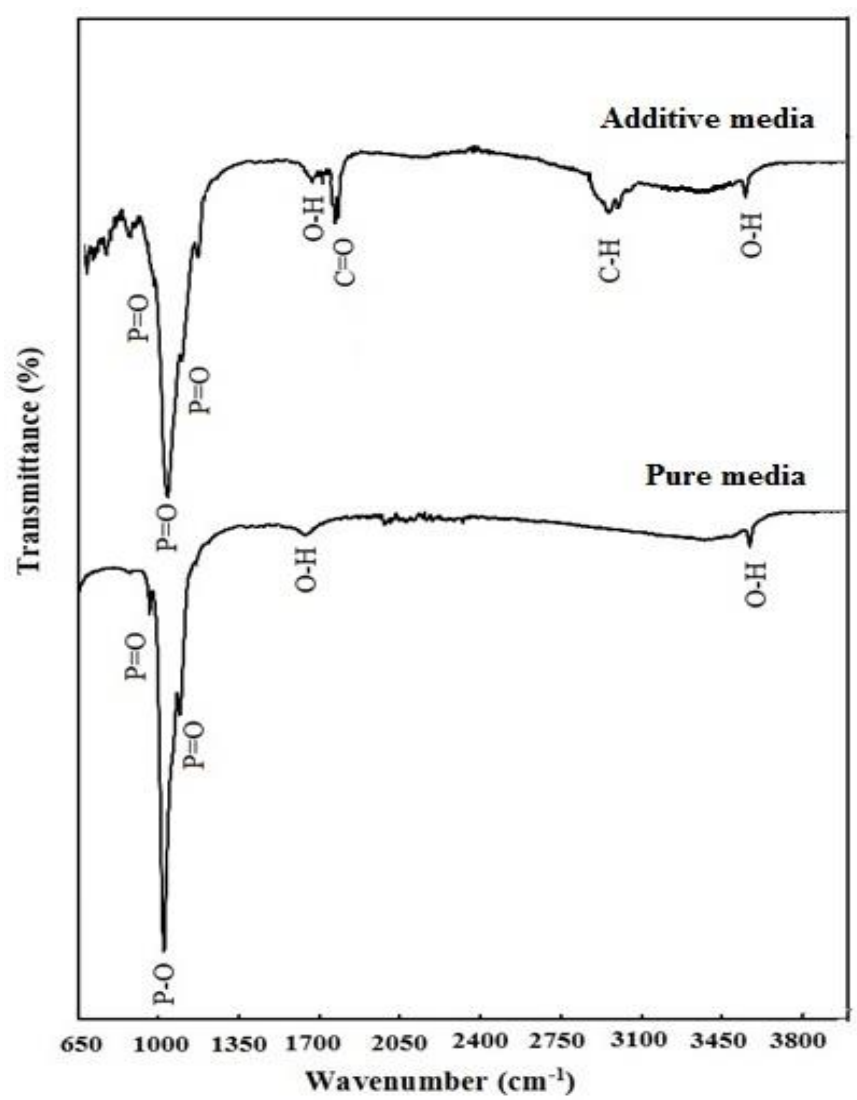

Figure 5. FTIR spectra of the crystals acquired (a) in pure solution and (b) solution containing 200 ppm butyric acid 
FTIR analysis showed that the crystals acquired in pure medium contain all the typical peaks of HAP $[21,22]$, in good agreement with the XRD results. The absorption peaks at $3570 \mathrm{~cm}^{-1}$ and $1641 \mathrm{~cm}^{-1}$ were related to the $\mathrm{O}-\mathrm{H}$ stretching vibration. The distinctive peak seen at $1021 \mathrm{~cm}^{-1}$ and the peak around $1090 \mathrm{~cm}^{-1}$ belonged to $\mathrm{P}=\mathrm{O}$ stretching vibrations and indicated the presence of the phosphate group in the structure. In addition, the peak observed at $960 \mathrm{~cm}^{-1}$ was also formed by the phosphate group. When the FTIR spectrum of the HAP crystals acquired in the $200 \mathrm{ppm}$ additive was examined, it was clearly seen that the crystals included the typical peaks of both HAP and butyric acid. The addition of butyric acid to the medium, new peaks were observed at about 3000 and $1710 \mathrm{~cm}^{-1}$. These peaks were attributed to the $\mathrm{C}=\mathrm{O}$ vibration of butyric acid [16]. In addition to the new peaks, the typical HAP peaks were slightly shifted in the presence of the additive. These results showed that the additive had bonded to the surface of the crystals.

\subsection{Thermal Characteristics of HAP Crystals}

The structural evolution and thermal stability of the HAP were investigated by TGA. The weight losstemperature graph of the HAP crystals obtained in pure medium is illustrated in Figure 6a. It can be seen that firstly degradation starts at $100{ }^{\circ} \mathrm{C}$ and ends at $500{ }^{\circ} \mathrm{C}$. The weight loss taking place in this temperature range is attributed to the loss of water from the HAP crystals. They contain two different types of water, namely, adsorbed water and lattice water [23]. A significant weight loss did not occur in the range of $500-700{ }^{\circ} \mathrm{C}$. Between 700 and $900{ }^{\circ} \mathrm{C}$, a significant weight loss of $3.4 \%$ was attributed to dehydroxylation degradation [24].
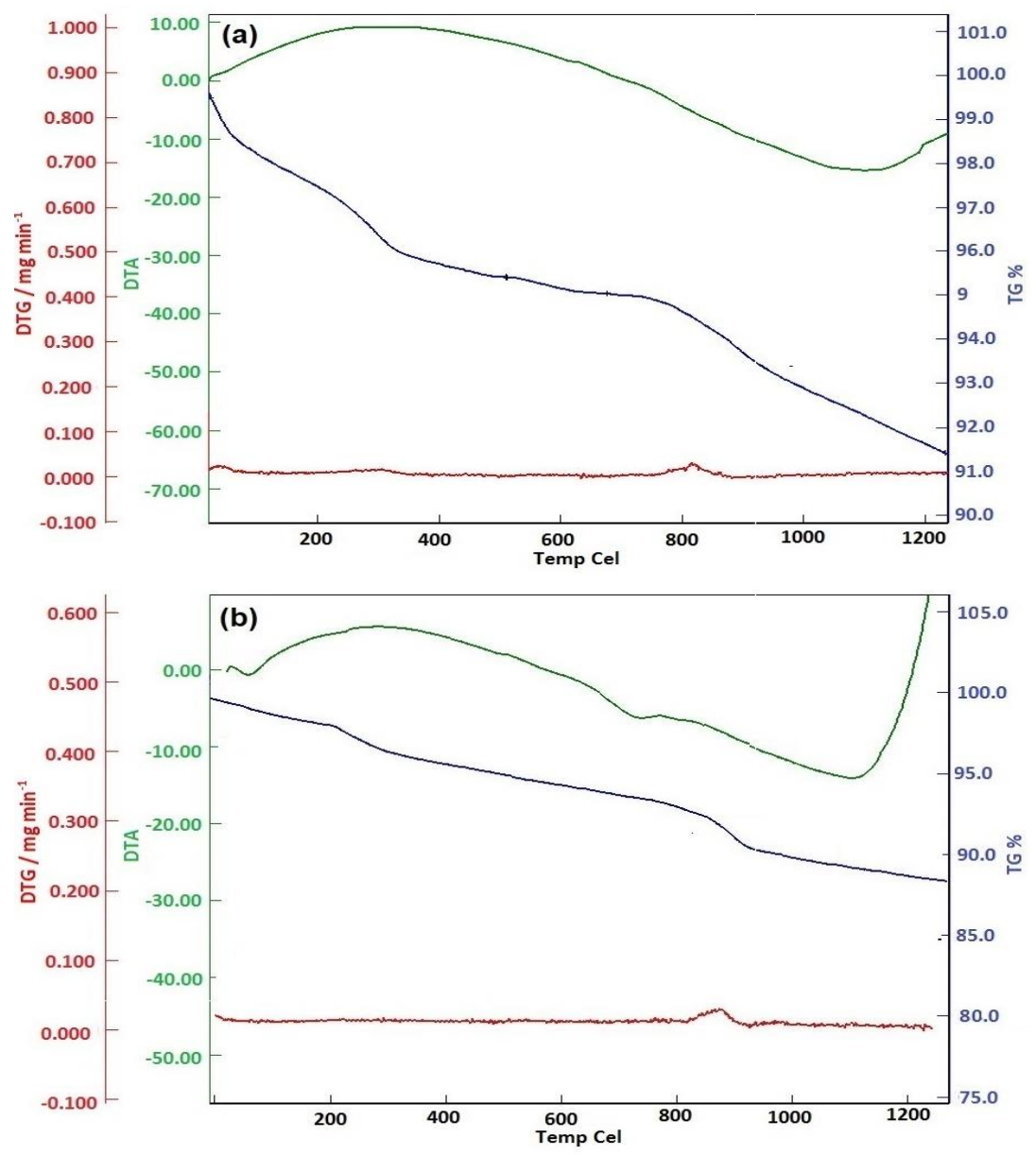

Figure 6. TG/DTG/DTA plots of the crystals acquired (a) in pure medium and (b) in the presence of $200 \mathrm{ppm}$ butyric acid. 
The TG/ DTG curves of the HAP crystals acquired in the presence of 200 ppm butyric acid are shown in Figure 6b. The same degradation trend as that observed in the crystals obtained in pure medium was found for the crystals obtained in additive medium. However, it was noticeable that the presence of butyric acid slightly affected the maximum peak temperature without changing the thermal profile. The maximum peak temperature for the HAP crystals without additive was $823{ }^{\circ} \mathrm{C}$ and this increased to $837^{\circ} \mathrm{C}$ for the crystals with $200 \mathrm{ppm}$ additive.

To gain more insight into the role of butyric acid in the thermal decomposition behavior, the average activation energy of the HAP crystals was analyzed using three models: distributed activation energy model (DAEM), Flynn-Wall-Ozawa (FWO) and Friedman.

\subsection{Evaluation of Various Kinetic Models}

The average activation energies for the thermal decomposition of the HAP crystals acquired in additive containing and non-additive media were calculated by using the TG data and DTG curves of the crystals for the conversion range of 0.2-0.9. The activation energies for the main thermal degradation zone were calculated in the temperature range of $700-900{ }^{\circ} \mathrm{C}$ where the most significant weight losses occurred. To determine the activation energy accurately, the DAEM model developed by Miura-Maki [25] was used in this study. The DAEM model was based on Eq. (1).

$$
\ln (\beta)=\ln \left(\frac{A E}{R g(x)}\right)-5.331-1.052 \frac{E}{R T}
$$

The commonly used FWO (Flynn-Wall-Ozawa) [26,27] and Friedman [28] iso-conversional models were also applied to the same data to compare the activation energy values that were calculated using the DAEM model and to analyze the consistency of the models. The FWO and Friedman methods do not require any prior assumption of a reaction model and their linearized final forms are given in Eq. (2) and Eq. (3), respectively.

$$
\begin{gathered}
\ln (\beta)=\ln \left(\frac{A E}{R g(x)}\right)-5.331-1.052 \frac{E}{R T} \\
\ln \left(\beta \frac{d x}{d T}\right)=\ln \left(\frac{d x}{d t}\right)=\ln [A f(x)]-\frac{E}{R T}
\end{gathered}
$$

Where $x$ is the conversion rate, $\beta$ is the heating rate, $A$ is the pre-exponential factor, $E$ is the activation energy, $R$ is the gas constant, $t$ is the time, $T$ is the absolute temperature, and $g(x)$ is the integrated reaction model. For a given value, it is possible to estimate the values of $E$ based on the gradients of the lines obtained by plotting $\ln \left(\beta / T^{2}\right)$ versus $1 / T, \ln (\beta)$ versus $1 / T$, and $\ln (d x / d t)$ versus $1 / T$ for the DAEM, FWO, and Friedman models, respectively.

Figure 7 shows the plots of the DAEM, FWO, and Friedman models for the HAP crystals acquired in pure solution. As can be seen from Figure 7, the individual lines were nearly parallel for all three models and all the models exhibited very good fitting. This parallelism behavior was associated with the single reaction mechanism owing to the dehydroxylation decomposition of the HAP crystals. All the models used provided accurate fits of the TG data and yielded acceptable results. The $\mathrm{R}^{2}$ values of all lines were higher than 0.95 , indicating the validity and reliability of the results. 
Polat / Eskişehir Tech. Univ. J. of Sci. and Technology A - Appl. Sci. and Eng. 20 (4) - 2019
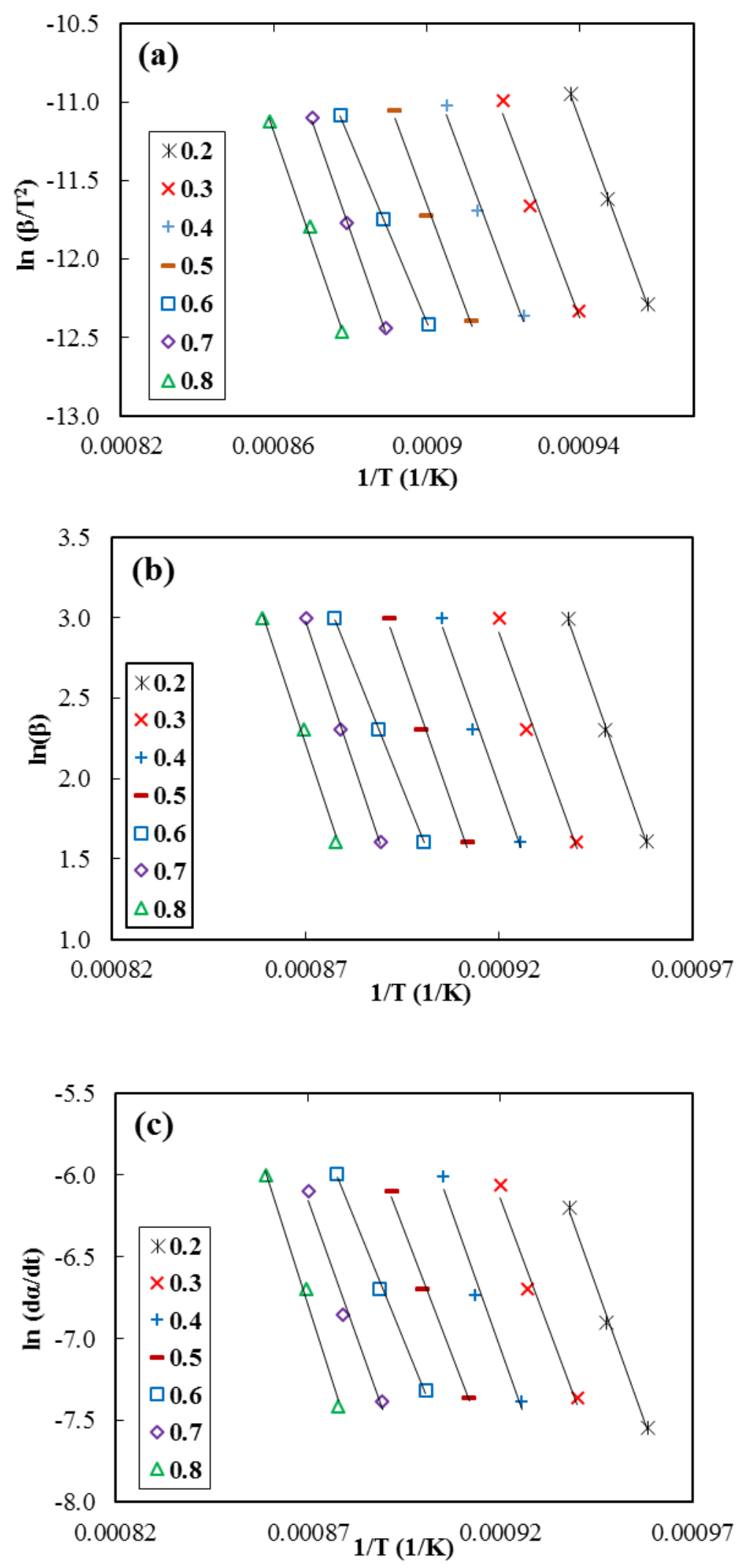

Figure 7. Plots of a) DAEM, b) FWO, and c) Friedman models for the HAP crystals acquired in pure medium 
The variations of activation energy according to conversion for the crystal acquired in pure and impure medium are illustrated in Figure 8.
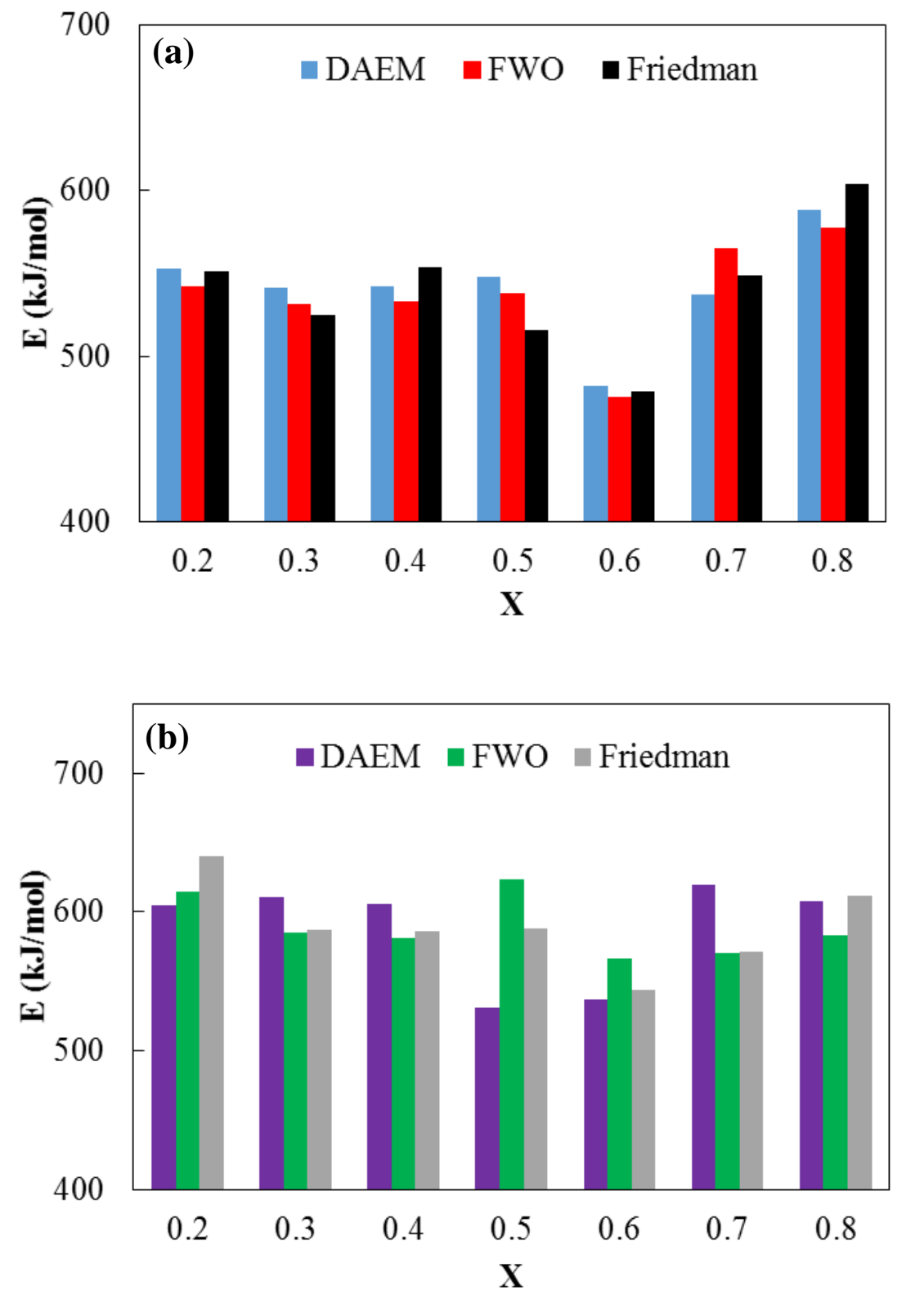

Figure 8. The variation of activation energy versus conversion degree for the crystals obtained (a) from pure medium and (b) from 200 ppm butyric acid containing media

The average activation energies for pure medium were determined as $538.8,537.6$, and $539.6 \mathrm{~kJ} / \mathrm{mol}$ using the DAEM, FWO, and Friedman models, respectively. These values were very close to each other and this indicated the accuracy of the results. The presence of butyric acid drastically affected the calculated activation energy. With the addition of the additive, the activation energies were in the range of 587-640 kJ/mol. The average activation energies for butyric acid medium were calculated as 588.2, 589.4, and $589.7 \mathrm{~kJ} / \mathrm{mol}$ using the DAEM, FWO, and Friedman models, respectively. The addition of butyric acid was accompanied by an increase in the activation energy. According to the calculated values of activation energy, the thermal decomposition of the crystals prepared with the additive required 
higher energy. Comparing with the activation energy for pure medium, the calculated values with respect to conversion degree were different for impure medium and a fluctuation was observed. The main reason behind this fluctuation trend is that the reaction mechanism was not the same as that in pure medium. The addition of the additive to the medium creates more complex reaction since the HAP crystals contain butyric acid.

\section{CONCLUSIONS}

The present work demonstrated the effects of butyric acid addition on HAP crystal characteristics and thermal decomposition kinetics. The XRD results for the crystals obtained in pure medium were consistent with the FTIR analysis and confirmed that the crystals were in typical HAP forms. The TEM analysis indicated that the surface morphology of the crystals changed when they were prepared in the presence of butyric acid. An increase in the additive concentration led to an increase in the dimensions of the crystals. The BET analysis results showed that the surface areas of the crystals acquired in the additive medium were lower than those of the crystals obtained in pure medium. Further characterization of the crystals with zeta potential analysis revealed that the surface charge of the crystals changed from $-20.9 \mathrm{mV}$ to $-13.9 \mathrm{mV}$. TGA analysis indicated that the crystals acquired in non-additive and additive medium underwent two degradation stages and differential thermal analysis confirmed the endothermic and exothermic pathways of these crystals. The addition of butyric acid shifted the maximum peak temperature to a higher value, but it did not affect the decomposition behaviour of the crystals. Thermal decomposition kinetics were examined using the DAEM, FWO, and Friedman models. The TGA data were well described by all the studied kinetic models and the activation energy changed with the addition of the additive. Compared to the pure medium, the average activation energy of the crystals increased from about 540 to $589 \mathrm{~kJ} / \mathrm{mol}$ in the presence of $200 \mathrm{ppm}$ additive owing to the adsorption of butyric acid on the HAP surface. In conclusion, the butyric acid can be potentially used as an additive for HAP crystallization to adjust the morphology, size, and thermal characteristics of the crystals.

\section{REFERENCES}

[1] Mann S. Molecular recognition in biomineralization. Nature 1988; 332: 119-124.

[2] Mann S. Biomineralization and biomimetic materials chemistry. J Mater Chem 1955; 935-946.

[3] Webster TH. Nanophase ceramics: The future orthopedic and dental implant material. Adv Chem Eng 2001; 27: 125-166.

[4] Yoshikawa H, Myoui A. Bone tissue engineering with porous hydroxyapatite ceramics. J Artif Organs 2005; 8: 131-136.

[5] Goodman SB, Yao Z, Keeney M, Yang F. The future of biologic coatings for orthopaedic implants. Biomaterials 2013; 34: 3174-3183.

[6] Neira IS, Kolen'ko YV, Lebedev OI, Tendeloo GV, Gupta HS, Guitian F, Yoshimura M. An effective morphology control of hydroxyapatite crystals via hydrothermal synthesis. Cryst Growth Des 2008; 9: 466-474.

[7] Habraken W, Habibovic P, Epple M, Bohner M. Calcium phosphates in biomedical applications: materials for the future?. Mater Today 2016; 19: 69-87.

[8] Canillas M, Pena P, Aza AH, Rodríguez MA, Calcium phosphates for biomedical applications. Boletín de la Sociedad Española de Cerámica y Vidrio 2017; 56: 91-112. 
[9] Koutsopoulos S, Dalas E. The effect of acidic amino acids on hydroxyapatite crystallization. J Cryst Growth 2000; 217: 410-415.

[10] Koutsopoulos S, Dalas E. Hydroxyapatite crystallization in the presence of serine, tyrosine and hydroxyproline amino acids with polar side groups. J Cryst Growth 2000; 216: 443-449.

[11] Koutsopoulos S, Dalas E. Hydroxyapatite crystallization in the presence of amino acids with uncharged polar side groups: glycine, cysteine, cystine, and glutamine. Langmuir 2001; 17: 10741079.

[12] Oner M, Dogan O. Inhibitory effect of polyelectrolytes on crystallization kinetics of hydroxyapatite. Prog Cryst Growth Charact Mater 2005; 50: 39-51.

[13] Mangood A, Malkaj P, Dalas E. Hydroxyapatite crystallization in the presence acetaminophe. J Cryst Growth 2006; 290: 565-570.

[14] Lima TARM, Brito NS, Peixoto JA, Valerio MEG. The incorporation of chromium (III) into hydroxyapatite crystals. Mater Lett 2015; 140: 187-191.

[15] Bucur AI, Bucur RA, Szabadai Z, Mosoarca C. Influence of small concentration addition of tartaric acid on the $220^{\circ} \mathrm{C}$ hydrothermal synthesis of hydroxyapatite. Mater Charact 2017; 132: 76-82.

[16] Yuan C, Liu B, Liu H. Characterization of butyric acid with different substitution patterns via FTIR, GC-MS, and TG-DTA. Carbohydr Polym 2015; 118: 36-40.

[17] Wada Y, Kobayashi T, Matsumoto M, Onoe K. A novel crystallization technique of hydroxyapatite utilizing contact reaction of minute droplet with atmospheric plasmas: Effects of the liquid source composition on the produced crystal properties. J Cryst Growth 2017; 475: 316-321.

[18] Wang Z, Xu Z, Zhao W, Sahai N. A potential mechanism for amino acid-controlled crystal growth of hydroxyapatite. J Mater Chem B 2015; 3: 9157-9167.

[19] Polat S, Sayan P. Characteristics and Thermal Kinetics of Hydroxyapatite Crystals Doped with Tricarballylic Acid. Chem Eng Technol 2018; 41: 1108-1117.

[20] Chen LJ, Mccrate JM, Lee JCM, Li H. The role of surface charge on the uptake and biocompatibility of hydroaxayapatite nanoparticles with osteoblast cells. Nanotechnology 2011; 22: 693-698.

[21] Hendi AA. Hydroxyapatite based nanocomposite ceramics. J Alloys and Compd 2017; 712: 147151.

[22] Tang B, Yuan H, Cheng L, Zhou X, Huang X, Li J, Effects of gallic acid on the morphology and growth of hydroxyapatite crystals. Arch Oral Biol 2015; 60: 167-173.

[23] Liao CJ, Lin FH, Chen KS. Thermal decomposition and reconstitution of hydroxyapatite in air atmosphere. Biomaterials 1999; 20: 1807-1813.

[24] Wang T, Dorner-Reisel A, Müller E. Thermogravimetric and thermokinetic investigation of the dehydroxylation of a hydroxyapatite powder. J Eur Ceram So 2004; 24: 693-698. 
[25] Miura K, Maki T. A simple method for estimating $f(E)$ and $k 0(E)$ in the distributed activation energy model. Energy Fuels 1998; 12: 864-869.

[26] Ozawa T. A new method of analyzing thermogravimetric data. Bulletin Chem Soc Japan 1965; 38: 1881-1886.

[27] Flynn JH, Wall LA. General treatment of the thermogravimetry of polymers. J Res Nat Bur Stand 1966; 70: 487-523.

[28] Friedman HL. Kinetics of thermal degradation of char-forming plastics from thermogravimetry. Application to a phenolic plastic. J Polym Sci 1964; 16: 183-195. 